OPEN ACCESS

Edited by:

Zhen-Ao Zhao,

Hebei North University, China

Reviewed by:

You Yu,

Soochow University, China

Juan Feng,

Peking University Health Science

Center, China

*Correspondence:

Weiqiang Huang

hwq2388@sina.com

Specialty section:

This article was submitted to

Cardiovascular Biologics and

Regenerative Medicine,

a section of the journal

Frontiers in Cardiovascular Medicine

Received: 13 May 2021

Accepted: 21 June 2021

Published: 08 July 2021

Citation:

Yuan Z and Huang W (2021) New Developments in Exosomal IncRNAs in Cardiovascular Diseases.

Front. Cardiovasc. Med. 8:709169.

doi: 10.3389/fcvm.2021.709169

\section{New Developments in Exosomal IncRNAs in Cardiovascular Diseases}

\author{
Zhu Yuan and Weiqiang Huang* \\ Department of Geriatric Cardiology, Guangxi Key Laboratory Base of Precision Medicine in Cardio-Cerebrovascular Diseases \\ Control and Prevention, Guangxi Clinical Research Center for Cardio-Cerebrovascular Diseases, The First Affiliated Hospital \\ of Guangxi Medical University, Nanning, China
}

Long non-coding RNAs (IncRNAs) are non-coding RNAs with lengths $>200$ nt and are involved in the occurrence and development of cardiovascular diseases (CVDs). Exosomes are secreted and produced by various cell types. Exosome contents include various ncRNAs, proteins and lipids. Exosomes are also important mediators of intercellular communication. The proportion of IncRNAs in exosomes is low, but increasing evidence suggests that exosomal IncRNAs play important roles in CVDs. We focused on research progress in exosomal IncRNAs in atherosclerosis, myocardial infarction, myocardial ischemia-reperfusion injury, cardiac angiogenesis, cardiac aging, rheumatic heart disease, and chronic kidney disease combined with CVD. The potential diagnostic and therapeutic effects of exosomal IncRNAs in CVDs are summarized based on preclinical studies involving animal and cell models and circulating exosomes in clinical patients. Finally, the challenges and possible prospects of exosomes and exosomal IncRNAs in clinical applications related to CVD are discussed.

Keywords: exosomal IncRNA, cardiovascular disease, atherosclerosis, myocardial infarction, cardiac
angiogenesis INTRODUCTION

Cardiovascular disease (CVD) is currently one of the main causes of death worldwide (1), and atherosclerosis and coronary heart disease are still representative challenges in CVD. In addition, the advent of an aging society has increased the cardiovascular burden, and the high incidence of rheumatic heart disease in developing countries and various concomitant cardiovascular diseases lead to final heart failure and other problems. The mainstream treatments, such as drugs, interventions, and surgery, cannot meet the needs of CVD patients with limited causes. In the case of myocardial infarction, emergency PCI or thrombolytic therapy can save the life of the patient but cannot block the adverse progression of the ischemic myocardium. For cancer patients, chemotherapeutic interventions can easily lead to premature heart failure (2). Therefore, exploring the molecular mechanisms underlying CVD and developing new treatments are critical for reducing CVD mortality.

LncRNAs are non-coding RNAs with lengths $>200 \mathrm{nt}$. These factors were once considered noise in the genome. With the development of high-throughput sequencing, the continuous identification of functional lncRNAs has changed the view of junk RNA $(3,4)$. LncRNAs are found in various cells and circulation and play key roles in cardiovascular development and CVDs, and lncRNAs are expected to become targets for the treatment of CVDs (5). LncRNAs are transcribed through various regulatory methods, and their interaction with microRNAs (miRNAs) has become a hot spot for studying the functional mechanism of lncRNAs (6). 
Exosomes are natural nanoscale cellular vesicles. The exosome surface is rich with specific marker proteins. After binding target cells, exosomes mainly introduce nucleic acids, proteins, and lipids through fusion with the plasma membrane and endocytosis. This content is delivered to the target cell for molecular regulation, representing an important mode of cellto-cell communication (7). Although the level of lncRNAs in exosomes is low, their role cannot be ignored. In recent years, many studies investigating exosomal lncRNAs provided new insight into crosstalk in the tumor microenvironment $(8,9)$, and research in the field of CVDs has begun to emerge. This review focuses on the relationship between exosomal lncRNAs and CVD, including the production of exosomal lncRNAs and their mechanism in CVD. Current studies have shown that lncRNAs derived from exosomes are mainly involved in posttranscriptional regulation by sponge adsorption of miRNAs. We hope that this review can provide a basis for their future clinical application through current research investigating exosomes and exosomal lncRNAs in CVD.

\section{LNCRNA CLASSIFICATION AND FUNCTION}

Similar to mRNAs, most lncRNAs are transcribed by RNA polymerase II (Pol II), capped at the $5^{\prime}$ end $(\mathrm{m} 7 \mathrm{G})$, tailed at the $3^{\prime}$ end (polyadenylation) and spliced, but lncRNAs lack an open reading frame and do not encode proteins for translation (10). According to the positional relationship between lncRNAs and protein-coding genes, lncRNAs can be divided into (1) sense lncRNAs that overlap with protein-coding genes; (2) antisense lncRNAs that are opposite to protein-coding genes; (3) lncRNAs from introns of protein-coding genes; (4) intergenic lncRNAs located between protein-coding genes; (5) lncRNAs derived from enhancers of protein-coding genes; (6) divergent lncRNAs transcribed from bidirectional promoters; and (7) circular RNAs (circRNAs) formed by the reverse splicing of protein-coding genes $(11,12)$. LncRNAs are located in the nucleus and cytoplasm and participate in the regulation of gene transcription through cis-acting or trans-acting mechanisms (10, 13-15). Before transcription, lncRNAs can bind proteins or RNAs for chromatin modification. For example, LNCPRESS1 interacts with SIRT6 to prevent the chromatin localization of SIRT6, maintain histone $\mathrm{H} 3 \mathrm{~K} 56$ and $\mathrm{H} 3 \mathrm{~K} 9$ acetylation, and activate transcription $(10,15,16)$. During transcription, lncRNAs can mediate gene silencing by interfering with the recruitment of transcription factors and Pol II to prevent transcription $(10,15)$. For example, the transcription of the $\ln \mathrm{RNA} A I R N$ can interfere with the recruitment of Pol $\mathrm{ll}$ and inhibit the transcription of LGF2R (17). LncRNAs can also form an R-loop triplet structure with DNA to complete the recruitment of transcriptional cofactors to the promoter, regulate chromatin accessibility, and promote transcription through lncRNAs transcribed by enhancers (10, 15). LncRNAs participate in regulation after transcription and translation. For example, lncRNAs bind miRNAs through a ceRNA mechanism that regulates target gene expression and interact with proteins to form IncRNA-protein complexes to regulate mRNA splicing and translation $(10,15)$.

\section{EXOSOME BIOLOGY}

Extracellular vesicles can be secreted by various cell types and are detected in body fluids. According to their biological mechanism and size, these vesicles are classified as exosomes, microcapsules, and apoptotic bodies. Exosomes are 30-150 nm in size; the plasma membrane initially invaginates to form early endosomes, and the endocytic membrane invades again to form late endosomes and multivesicular bodies (MVBs) (18). Studies have shown that there are two mechanisms underlying the formation of intraluminal vesicles (ILVs) in MVBs as follows: an ESCRT-dependent pathway and an ESCRTindependent pathway. The ESCRT complex includes ESCRT-0, ESCRT-I, ESCRT-II, ESCRT-III and the auxiliary proteins Alix and Vps4 $(18,19)$. ESCRT-0 recognizes ubiquitinated proteins outside the endosomal membrane. The complete recruitment of ESCRT-I and ESCRT-II occurs under the stimulation of phosphatidylinositol 3-phosphate (PIP3), hepatocyte growth factor-regulated tyrosine kinase substrate (HRS), ubiquitination of the cytosolic tails of endocytic proteins, or curved membrane topology. ESCRT-I binds the ubiquitinated cargo to the endosomal membrane and cooperates with ESCRT-II to open the endoluminal membrane for germination. ESCRT-III participates in the membrane rupture of the ILV neck under the recruitment of Alix, binds TSG101 and participates in the formation of the ESCRT-I complex. Under the action of a deubiquitinating enzyme, the ubiquitin mark is removed from the carrier protein to complete the sorting process. ESCRT-III is decomposed and recycled by the AAA-ATPase suppressor of potassium transport growth defect-1 (SKD1) for the next round of cargo recruitment (18-20). Studies have shown that ILVs can be formed in the absence of the ESCRT complex, indicating that the biological behavior of MVBs can be independent of the ESCRT pathway (21). For example, the lipid raft microdomains formed by ceramide and tetraspanin microdomains formed by CD63 trigger the transport of ILVs to $\operatorname{MVBs}(19,20)$. Usually, MVBs are degraded by fusion with lysosomes after ISG-mediated modification of the TSG101 protein or are fused with the plasma membrane under the coordinated control of the cytoskeleton (microtubules and actin), soluble NSF attachment protein receptor (SNARE), and Rab GTPase to release exosomes (19) (Figure 1). A layer-by-layer analysis of the biological behavior of exosomes suggests that exosomes have great potential as a new type of biomarker for prediction and diagnosis in the field of cancer (22). Regarding CVD, exosomes have been reported to have potential application in the diagnosis and treatment of adriamycininduced cardiotoxicity (23). In addition, Ghafarian et al. discussed the basic science and clinical application of exosomes in CVD (24).

\section{EXOSOMAL LNCRNA SORTING AND MECHANISM OF ACTION}

The mechanism by which lncRNAs are sorted into exosomes is still unclear. However, RNA-binding proteins (RBPs), including the hnRNP family [hnRNPA2B1 (25, 26), hnRNPG (27), 


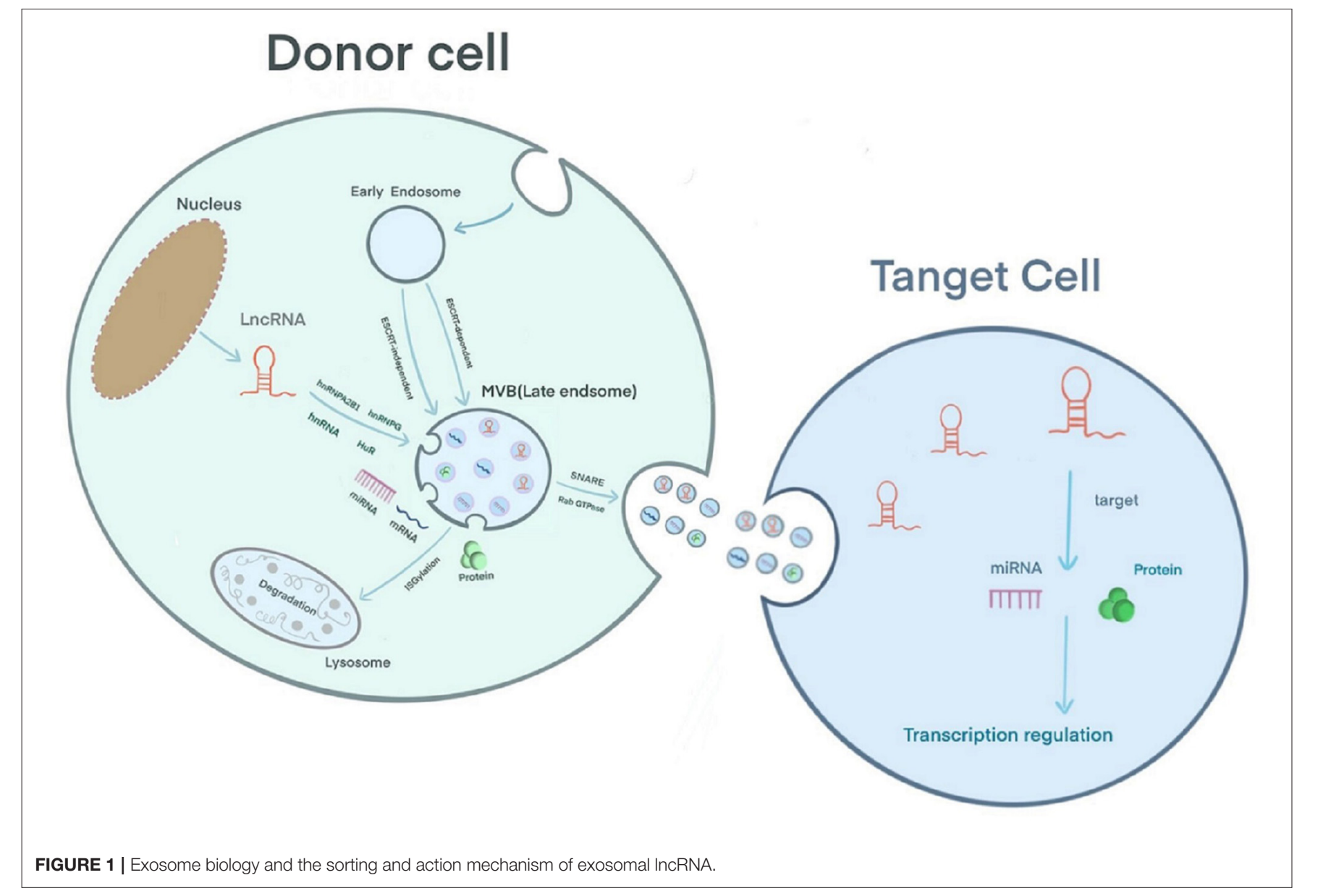

hnRNPK (28)] and human antigen R (HuR) (29), have been confirmed to participate in the sorting of lncRNAs into exosomes (30). Furthermore, in sunitinib-resistant kidney cancer cells, hnRNPA2B1 has been shown to bind the $5^{\prime}$ end of LNCARSR, and knocking down the level of hnRNPA2B1 or mutating the binding site of hnRNPA2B1 reduces the level of $L N C A R S R$ in exosomes, suggesting that hnRNPA2B1 specifically facilitates LNCARSR loading into exosomes (31) (Figure 1). Previously, Zheng et al. introduced the mechanism of action of exosomal miRNAs, i.e., exosomal miRNAs affect the progression of CVD by binding target genes and mediating target mRNA silencing (32). The difference is that exosomal lncRNAs can be used for disease regulation through epigenetic modification. Furthermore, exosomal lncRNAs often act as sponges of endogenous miRNAs, mediating the expression of their target genes or activating related signaling pathways to produce biological effects. For example, Zang et al. found that the combination of the lncRNAs UFC1 and EZH2 resulted in $\mathrm{H} 3 \mathrm{~K} 27$ trimethylation and PTEN expression inhibition, which ultimately promoted the progression of non-small cell lung cancer (33). Zhuo et al. found that the exosomal lncRNA FAM138B derived from cancer cells can alleviate the progression of hepatocellular carcinoma by sponging $M I R-$ 765 (34).

\section{EXOSOMAL LNCRNA IN CARDIOVASCULAR DISEASE}

In recent years, research investigating exosomal lncRNAs has become a trend. Especially in the field of cancer, several studies have shown that exosomal lncRNAs may become new biomarkers of and targets for cancer progression and treatment (35). In addition, similar studies investigated exosomal lncRNAs in various systemic diseases. For example, exosomal lncRNAs are expected to become a therapeutic target for lung diseases (36). In the field of CVD, Wang et al. found that the release of the exo-circRNA HIPK3 from hypoxic cultured cardiomyocytes protects against cardiac microvascular endothelial oxidative damage by targeting the MIR29A/IGF-1 pathway and promotes the proliferation and migration of cardiac endothelial cells through the MIR29a/VEGF axis $(37,38)$. Ni et al. discussed the relationship between exocrine-derived non-coding RNAs (lncRNAs and miRNAs) in endothelial cells and vascular smooth muscle cells and their mechanism in vascular senescence (39). Recently, Cheng et al. found in vitro that the exo-lncRNA ZEB1AS1 secreted by human umbilical vein endothelial cells induced by oxidized low-density lipoprotein (ox-LDL) enhances the damage caused by the MIR-590-5P/ETS1 axis to human umbilical vein endothelial cells through the TGF- $\beta /$ Smad pathway (40). 
TABLE 1 | Progress of exosomal IncRNA in preclinical study of CVD.

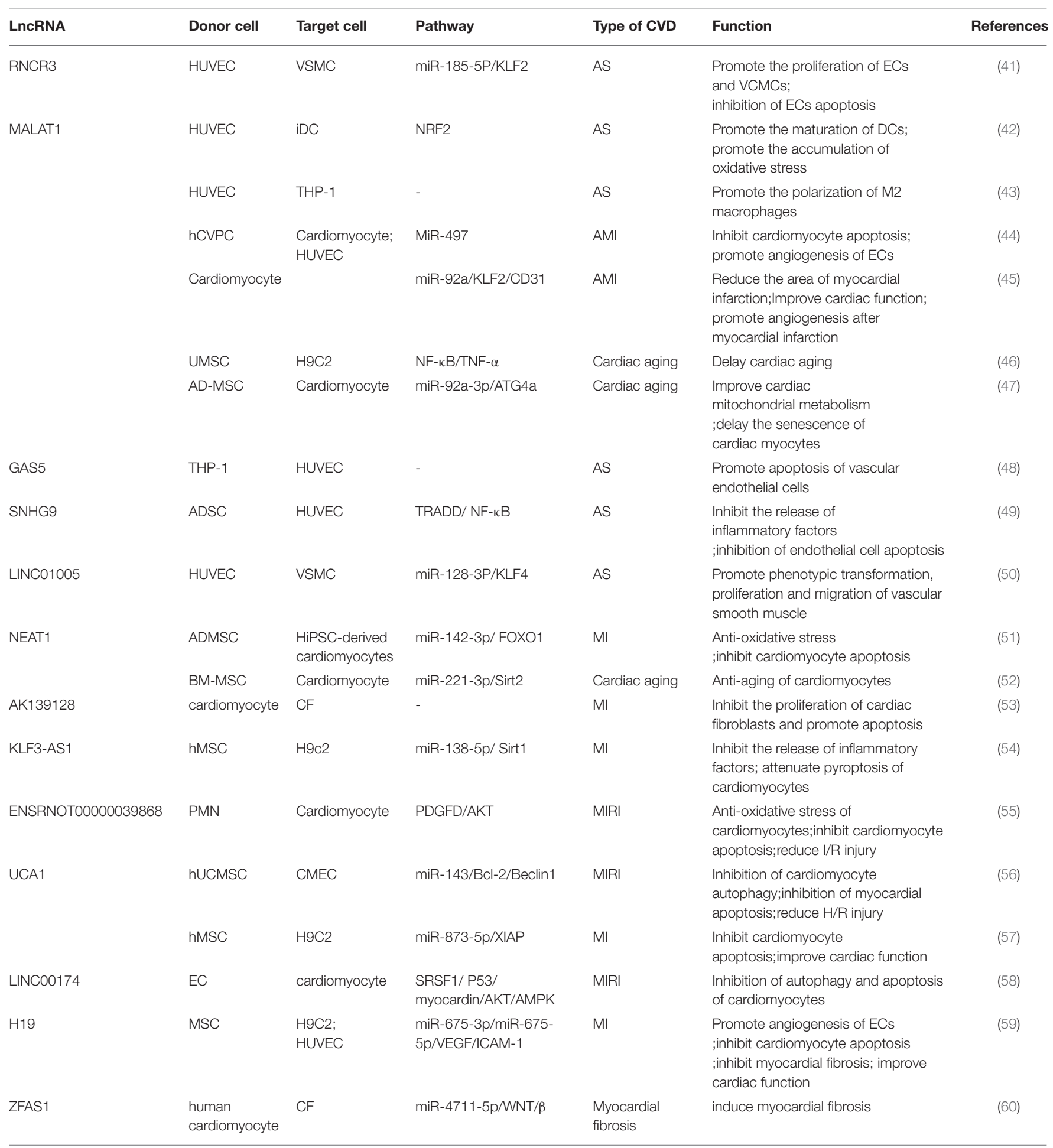

Current studies have shown that exosomal lncRNAs participate in the dynamic evolution of underlying cardiovascular diseases through various pathways, involving all aspects of their pathophysiology and potential treatment. For example, lncRNAs participate in the initial development of atherosclerosis, the occurrence of acute myocardial infarction (AMI) and ischemia-reperfusion injury, cardiac angiogenesis, repair and protection against cardiac aging (Table 1 and Figure 2). The differential changes in lncRNAs in patient plasma exosomes make them potential 


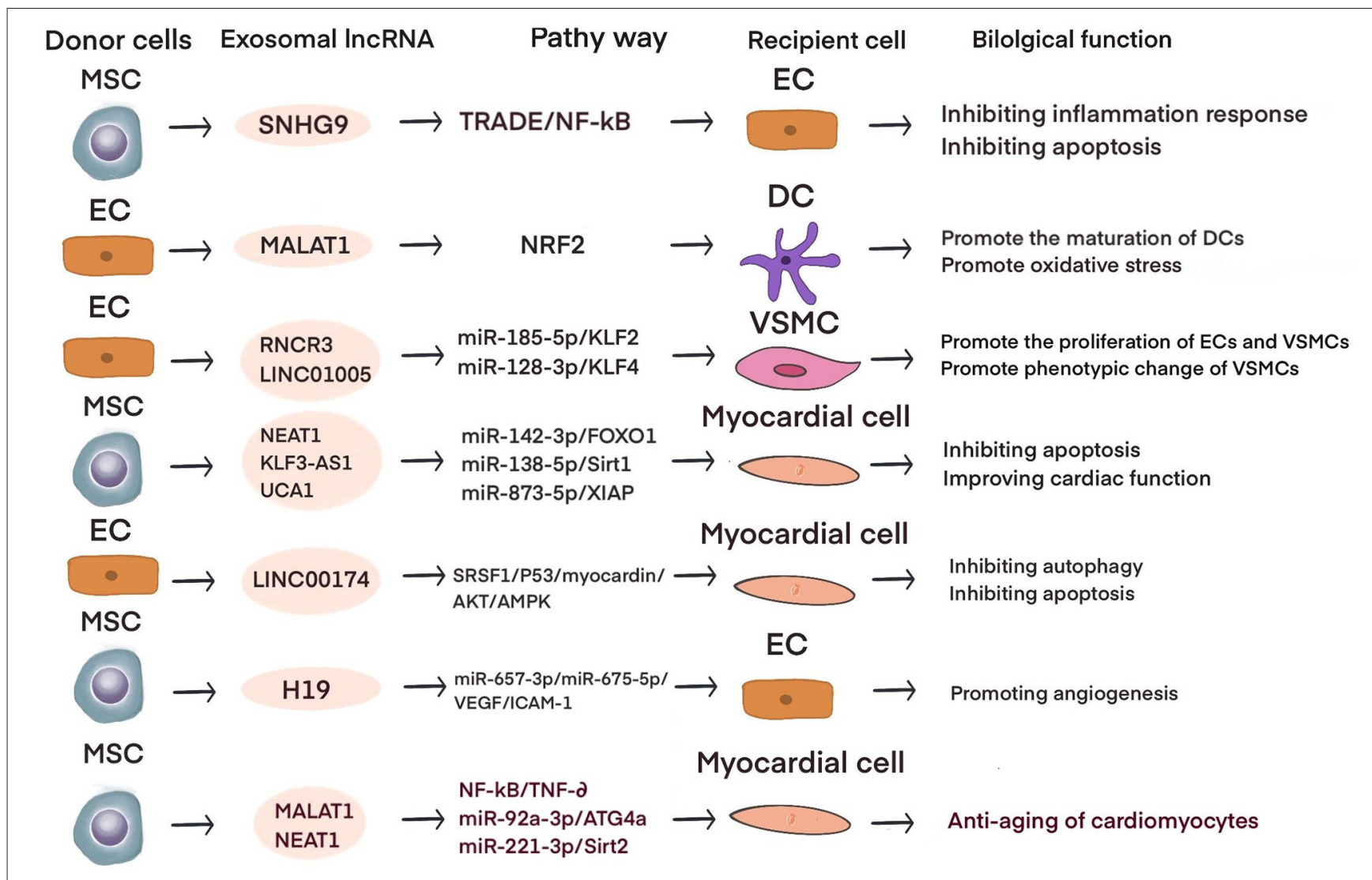

FIGURE 2 | The role of exosomal IncRNA in preclinical studies of cardiovascular diseases.

TABLE 2 | The role of serum exosomal IncRNA in cardiovascular disease.

\begin{tabular}{lllll}
\hline Exosomal IncRNA & Expression & Disease & Function & References \\
\hline HIF1a-AS1 & Up & AS & Diagnostic & $(61)$ \\
NEAT1 & Up & STEMl & Diagnostic & $(62)$ \\
ENST00000556899.1; & Up & AMl & Prognostic & $(63)$ \\
ENST00000575985.1 & & & & \\
UCA1 & Up & AMl & Diagnostic & $(57)$ \\
SOCS2-AS1 & Down & CAD & Diagnostic & $(64)$ \\
RP13-820C6.2 & Down & RHD & Diagnostic; & $(65)$ \\
RP11-339B21.15 & & & Therapeutic & \\
G004800 & & & & \\
XLOC_010028 & & & & \\
XLOC_004201 & Up & & & \\
\hline
\end{tabular}

biomarkers for the diagnosis and treatment of CVDs (Table 2 and Figure 3).

\section{ATHEROSCLEROSIS}

Atherosclerosis (AS) is the most common type of CVD and the root cause of myocardial infarction. The main risk factors are aging, hyperlipidemia, obesity, hypertension, and smoking. Endothelial injury is considered the first step in the development of atherosclerosis. Subsequently, after inflammatory cell infiltration, vascular smooth muscle cells (VSMCs) proliferate and activate, exacerbating plaque formation. Therefore, targeting endothelial dysfunction, inflammatory cells and the phenotypic transition of VSMCs are critical for the study of the development and treatment of atherosclerosis. For example, exo-MIR143/MIR145 secreted by endothelial cells expressing KLF2 can communicate with smooth muscle cells, increase the expression of dedifferentiation-related genes, and reduce atherosclerosis in ApoE-/- mice (66). Macrophagederived exo-MIR99A/MIR146B/MIR378A targets NF-кB/TNF$\alpha$ signaling to inhibit inflammation, promote macrophage M2 polarization, and reduce the area of atherosclerotic necrosis (67).

Obesity is a risk factor for atherosclerosis; obesity promotes endothelial cell (EC) dysfunction, accelerates endothelial cell apoptosis and the release of inflammation, and promotes the development of atherosclerosis $(68,69)$. Exosomal lncRNAs are involved in endothelial dysfunction. For example, lncRNA SNHG9 overexpression in adipose stem cell-derived exosomes can inhibit endothelial cell apoptosis, inhibit NF- $\kappa \mathrm{B}$ signaling, and reduce inflammation. TRADD, which is the target gene of SNHG9, is negatively associated with SNHG9 and inversely regulates the protective effects of SNHG9 to inhibit EC disorders (49). 


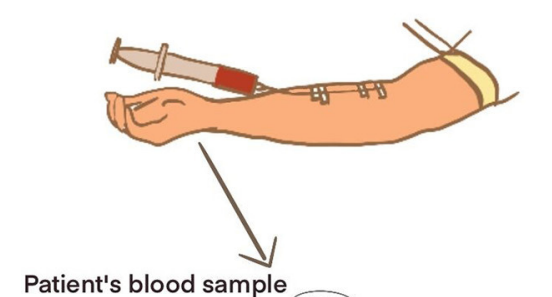

Patient's blood sample and identification of exosomal IncRNA

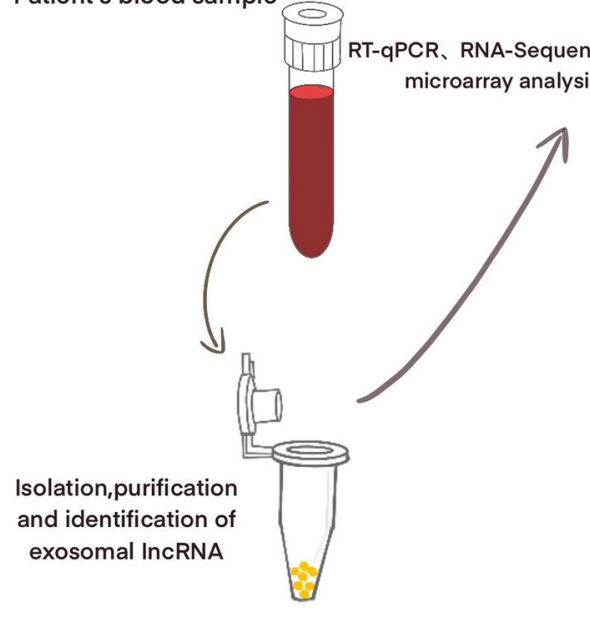

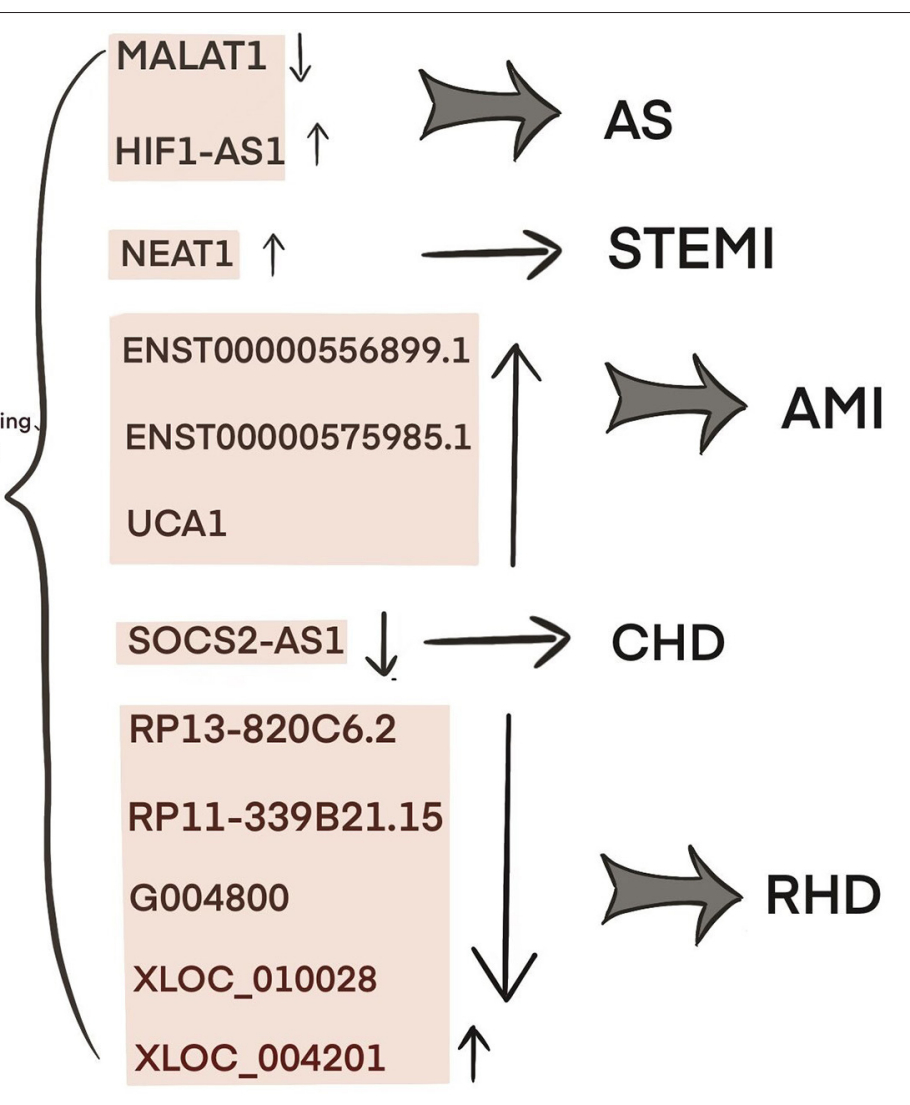

FIGURE 3 | Exosomal IncRNA in the serum of CVD patients as potential biomarkers.

M2 macrophages can fight the progression of atherosclerosis (67). Exosomal lncRNAs mediate communication between ECs and immune cells. For example, Huang et al. found that the expression level of the lncRNA MALAT1 was significantly increased in exosomes secreted by ox-LDL-stimulated human umbilical vein endothelial cells (HUVECs) and that coculture with these exosomes increased THP-1 cell expression of MALAT1 and promoted the polarization of M2 macrophages (43). A study by Chen et al. showed that the expression of the lncRNA GAS5 in exosomes derived from THP-1 cells under ox-LDL stimulation was significantly upregulated. Exosomes secreted by ox-LDL-stimulated THP-1 cells with GAS5 knockout were cocultured with HUVECs, and PHK67labeled exosomes were internalized by HUVECs and significantly reduced HUVEC apoptosis. In contrast, exosomes secreted by ox-LDL-stimulated GAS5-overexpressing THP-1 cells could accelerate HUVEC apoptosis (48). Hong et al. found that the expression of MALAT1 was significantly reduced in the serum of patients with atherosclerosis and exosomes derived from ox-LDL-stimulated HUVECs. Exosomes derived from oxLDL-stimulated HUVECs overexpressing MALAT1 are taken up by dendritic cells (DCs) and can inhibit DC maturation, activate NRF2 signaling, and inhibit reactive oxygen species (ROS) accumulation in DCs. Silencing MALAT1 results in the opposite effects. Similarly, in a mouse model of atherosclerosis,
ox-LDL-stimulated exosomes exacerbated atherosclerosis in mice. This finding shows that the expression of MALAT1 is decreased in vascular endothelial cell-derived exosomes induced by ox-LDL, inhibits NRF2 signaling, leads to ROS accumulation and DC maturation, and accelerates the development of atherosclerosis (42).

Exosomal lncRNAs mediate the interaction between endothelial cells and VSMCs. For example, Shan et al. found that apoE-/- mice expressed increased levels of the lncRNA RNCR3 during the atherosclerotic stage, and RNA fluorescence in situ hybridization revealed RNCR3 localization in endothelial cells and arterial smooth muscle cells. After knocking out RNCR3 in vivo, the endothelial cell coverage and smooth muscle proliferation were significantly reduced. In vitro cell experiments showed that after knocking out RNCR3, HUVEC proliferation slowed, and apoptosis accelerated. After coculturing HUVEC-derived exosomes (HUVEC-exos) with VSMCs, VSMC proliferation and migration were inhibited. Biological analyses and luciferase assays show that HUVECexo-RNCR3 targets the MIR-185-5P/KLF2 axis to promote the proliferation of ECs and VSMCs and plays a protective role in the development of atherosclerosis (41). Zhang found that coculturing ox-LDL-stimulated HUVECs-exo-LINC01005 with VSMCs significantly downregulated the smooth muscle cell contraction markers $\alpha$-SMA and SM22a and upregulated the 
VSMC proliferation marker OPN, indicating that HUVECsexo-LINC01005 promoted phenotypic changes in VSMCs. The database and luciferase reporter assay show that HUVECs-exoLNC01005 mediates phenotypic changes in endothelial cells and VSMC proliferation through the MIR-128-3P/KLF4 axis (50). It is suggested that the communication of exosomal lncRNAs among ECs, VSMCs and immune cells is mainly involved in the progression of atherosclerosis by targeting miRNAs or directly regulating the expression of genes.

The differences in the expression of exosomal lncRNAs in peripheral circulation in patients with atherosclerosis may represent a potential biomarker for the diagnosis of atherosclerosis. For example, Song et al. found significantly low expression of exosomal SNHG9 in the plasma of obese patients and revealed that this level was associated with endothelial dysfunction (49). Hong et al. found that a low expression of exosomal MALAT1 in the serum of patients with atherosclerosis led to DC maturation and promoted the progression of atherosclerosis (42). Wang et al. examined 65 blood samples from atherosclerosis patients and 68 blood samples from healthy volunteers and found that the blood exosomal concentration and exo-lncRNA HIF1A-AS1 expression in the atherosclerosis patients were significantly higher than those in the healthy group. A receiver operating characteristic (ROC) curve analysis showed that the areas under the curve of the plasma exosomes and exo-HIF1A-AS1 were 0.856 and 0.823 , respectively. This finding indicates that plasma exo-HIF1A-AS1 may be a potential biomarker for the diagnosis of atherosclerosis (61). It is suggested that the differential expression of serum exosomal lncRNAs in patients with atherosclerosis may play an auxiliary role in diagnosis.

\section{MYOCARDIAL INFARCTION}

Myocardial infarction is caused by coronary artery stenosis or occlusion, and the myocardium is in a state of ischemia and hypoxia. Apoptosis and fibrosis of ischemic and hypoxic cardiomyocytes lead to ventricular remodeling, which, in turn, causes arrhythmia and heart failure. Since the 21st century, the performance of cell therapy in the treatment of myocardial regeneration has been unsatisfactory. The paracrine mechanism that plays a major role in cell therapy has given high hopes regarding the use of exosomes characterized by non-cellular therapy (70-74). For example, in a mouse model of myocardial infarction, exosomes derived from mouse embryonic stem cells promote survival and proliferation in cardiac progenitor cells by delivering miR-294 (75). Intramuscular injection of exosomes derived from cardiosphere-derived cells can reduce acute and chronic myocardial infarction injury in pigs, reduce the area of myocardial infarction, reduce the area of scars, and combat cardiac remodeling (76).

In addition to exosomal miRNAs, mesenchymal stem cell (MSC)-derived exosomal lncRNAs also play important roles in myocardial infarction. For example, Che et al. found that the exosomal lncRNA NEAT1 secreted by migration inhibitory factor (MIF)-induced human adipose mesenchymal stem cells
(ADMSCs) inhibits $\mathrm{H} 2 \mathrm{O} 2$-induced cardiomyocyte apoptosis. The database and dual luciferase gene reports show that the ADMSC $^{\mathrm{MIF}}$-derived exosomal NEAT1 reduces $\mathrm{H} 2 \mathrm{O} 2$-induced oxidative stress in cardiomyocytes and reduces cardiomyocyte apoptosis through the MIR-142-3p/FOXO1 axis (51). Mao et al. detected the IncRNA KLF3-AS1 in exosomes secreted by human MSCs (hMSCs). Injection of hMSC-exos in a rat myocardial infarction model significantly reduced the expression of the proinflammatory factors IL- $1 \beta$ and IL-18 in cardiomyocytes and the expression of NLRP3, Asc, and Caspase-1, indicating that hMSC-exos induced cardiomyocyte pyrolysis. In an in vitro hypoxic cardiomyocyte model, the protective effect of hMSCexos was consistent with that observed in the in vivo experiments. Database and dual luciferase gene reporter experiments showed that the hMSC-exo-KLF3-AS1/MIR-138-5p/SIRT1 axis mediates protection in hypoxic cardiomyocytes (54). Sun et al. performed cell and animal experiments and showed that the exosomal lncRNA UCA1 secreted by hypoxia-induced human bone marrow MSCs (BMSCs) sponges MIR-873-5p, regulates XIAP, and enhances AMPK phosphorylation in cardiomyocytes. This lncRNA also increases the expression of antiapoptotic proteins, such as p53, bax, and cleaved caspase-3, and inhibits the expression of the apoptotic protein bcl-2 (57).

Studies have shown that exosomal lncRNAs mediate communication between cardiomyocytes and cardiac fibroblasts (CFs) in the context of myocardial infarction. For example, Wang et al. found that coculturing hypoxic cardiomyocyte-derived exosomes and CFs significantly inhibited CF proliferation, migration and invasion and promoted apoptosis. An RNA-seq analysis revealed the differential expression of the IncRNA $\mathrm{AK} 139128$. In vivo experiments showed that exo-AK139128 exacerbated CF apoptosis in rats with myocardial infarction. Western blot analyses showed that the expression of the apoptotic protein bcl-2 increased, while the expression of the antiapoptotic protein bax decreased. This finding shows that exosomal AK139128 secreted by hypoxic cardiomyocytes promotes CF apoptosis (53). The above studies show that in addition to exosomal lncRNAs derived from mesenchymal stem cells, which are expected to become therapeutic targets for the treatment of infarcted myocardium, exosomal lncRNAs between cardiac cells also have potential therapeutic significance and research value.

Recent studies have shown that significant differences exist in plasma exosomal lncRNAs in patients with myocardial infarction, suggesting that these factors may have an auxiliary diagnostic effect. For example, Chen et al. found that the serum expression of exosomal NEAT1 and MMP9 in patients with ST-segment elevation myocardial infarction (STEMI) was significantly higher than that in patients with unstable angina and non-myocardial infarction, while the level of MIR204 was relatively lower. Spearman tested the correlation among the three factors and found that NEAT1 was positively correlated with the MMP9 levels, but NEAT1 was not significantly correlated with MIR204, MIR204, or MMP9. A logistic regression analysis revealed that NEAT1, MIR204, and MMP9 are independent predictors of STEMI (62). Zheng et al. found that ENST00000556899.1 and ENST00000575985.1 were 
significantly upregulated in circulating exosomes from AMI patients and control patients. An ROC analysis showed that ENST00000556899.1 and ENST00000575985.1 have areas under the myocardial infarction curve of 0.661 and 0.751 , respectively, and are positively correlated with inflammatory biomarkers, myocardial infarction prognostic indicators, and myocardial injury markers (63). Sun et al. showed that the plasma expression of the exosomal IncRNA UCA1 in patients with myocardial infarction was increased. An ROC analysis showed that human plasma exosomal UCA1 may be a non-invasive biomarker for the diagnosis of AMI (57). Liang et al. enrolled 227 subjects and divided them into a coronary heart disease group, an early coronary heart disease group, and a normal coronary artery control group. Gene chip detection and RT-PCR showed that the exo-lncRNA SOCS2-AS1 was significantly downregulated in the coronary heart disease group. A Pearson correlation analysis showed that the plasma exo-SOCS2-AS1 levels were negatively correlated with the LPA and PLT levels. Univariate and multivariate logistic regression analyses showed that the plasma exosomal SOCS2-AS1 levels were an independent protective factor for coronary heart disease (64). These data suggest that exosomal lncRNAs may play an auxiliary role in the prediction and diagnosis of patients with myocardial infarction.

\section{MYOCARDIAL ISCHEMIA-REPERFUSION INJURY}

Myocardial ischemia-reperfusion injury (MIRI) refers to the pathological process during which the ischemic myocardium returns to normal perfusion after a coronary artery is partially or completely occluded, which, in turn, exacerbates myocardial injury. In clinical practice, MIRI is common in patients with myocardial infarction after emergency percutaneous coronary intervention (PCI) or thrombolytic therapy (77). The potential molecular mechanisms involved include oxidative stress, intracellular calcium ion overload, rapid recovery of $\mathrm{PH}$, opening of the mitochondrial permeability transition pore (MPTP), the inflammatory response, and late myocardial reperfusion injury $(77,78)$. In recent years, treatments for MIRI have included ischemic preconditioning (IPC), ischemic postprocessing (IPost), remote ischemic conditioning (IPC), the use of drugs to prevent myocardial reperfusion injury, therapeutic hyperbaric oxygen (HBO) and hypothermia (77-79). Recent studies have shown that the cardioprotective effect of IPC is mediated by exosomes (80). For example, exosomes derived from rat fibroblasts can protect cardiomyocytes after ischemia by targeting MIR-423-3p/RAP2C to reduce MIRI (81).

Similarly, exosomal UCA1 derived from hMSCs was discovered by Sun et al. to exert myocardial protection after myocardial infarction (57). Diao et al. found that exosomes derived from human umbilical cord blood MSCs (hUCMSCs) could inhibit hypoxia/reoxygenation (H/R)-induced cardiac microvascular endothelial cell (CMEC) apoptosis in SpragueDawley rats. In addition, hUCMSC-exos can inhibit macrophage autophagy under hypoxia. In an in vivo IR rat model, injection of hUCMSC-exos improved the ultrastructure of rat CMECs, inhibited apoptosis, and reduced the vascular endothelial injury markers TM and vWF. An RT-qPCR analysis verified that hUCMSC-exos were enriched with UCA1. In addition, dual luciferase reporter and RNA pull-down experiments showed that hUCMSC-exo-UCA1 inhibited autophagy and apoptosis in H/Rinjured rat CMECs by regulating the miR143/BCL-2/BECLIN1 axis (56).

Recent studies have shown that exosomal lncRNAs are involved in the communication between polymorphonuclear cells (PMNs) and IR cardiomyocytes. For example, Zhai et al. used an H/R cardiomyocyte model and showed that PMN-exos stimulated by the calcium sensitive receptor (CaSR) activator cinacalcet were taken up by cardiomyocytes, increased the expression of $\mathrm{p}-\mathrm{AKT}$ and $\mathrm{Bcl}-\mathrm{xL}$, reduced the production of NOX2 and ROS, and reduced cardiomyocyte apoptosis, while the AKT inhibitor HY15186 could reverse these effects. A high-throughput sequencing analysis of CaSR-PMN-exos found differential expression of the lncRNA ENSRNOT00000039868 (lncRNA 39868) and predicted PDGFD as the target protein of lncRNA 39868. siRNA-mediated silencing of the lncRNA 39868 significantly reduced PDGFD mRNA expression. In vivo experiments showed that after intravenous injection of CaSRPMN-exos via the tail vein in rats, the area of myocardial infarction and MDA secretion were significantly reduced, the SOD levels were increased, and cardiac function was improved. These results suggest that CaSR-PMN-exosomal lncRNA 39868 upregulates the protein PDGFD, regulates these effects through the AKT pathway, and reduces MIRI (55).

Exosomal lncRNAs derived from endothelial cells have protective effects on IR cardiomyocytes. For example, $\mathrm{Su}$ et al. measured the expression of exosomal LINC00174 derived from vascular endothelial cells. After coculturing H/R cardiomyocytes with exo-LINC00174 in vitro, P53 was inhibited, and P53induced autophagy enhancement and cardiomyocyte apoptosis were inhibited. In contrast, the expression of SRSF1 was increased. RNA pull-down experiments and RIP verified the binding of LINC00174 to SRSF1. In I/R mice, AKT/AMPK phosphorylation was increased, indicating that myocardial autophagy was activated, but P53 knockout inhibited autophagy activation and cardiomyocyte apoptosis. A dual luciferase reporter assay confirmed that MYOCARDIN was the binding site of P53. Vascular endothelial cell exo-LNC00174 bound SRSF1 to inhibit P53 expression, MYOCARDIN transcription, AKT/AMPK activation, and ultimately autophagy activation and apoptosis in I/R cardiomyocytes (58). These results suggest that the mechanism of cardiomyocyte protection mediated by exosomal lncRNAs in myocardial ischemia-reperfusion injury involves crosstalk among mesenchymal stem cells, polymorphonuclear cells and endothelial cells. The study of exosomal lncRNAs in myocardial ischemia-reperfusion still needs more exploration.

\section{CARDIAC ANGIOGENESIS}

Currently, revascularization treatment for AMI or ischemic cardiomyopathy mainly involves PCI, coronary artery bypass 
grafting and thrombolysis to restore the myocardial blood supply and save the dying myocardium. However, MIRI follows, and for patients who cannot undergo surgery, there is an urgent need for a new alternative therapy. In addition to cell therapy to rescue and restore cardiomyocytes, increasing research suggests that promoting the survival of cardiac endothelial cells, treating cardiac angiogenesis, and mediating the recanalization of cardiac collaterals have great therapeutic potential for the treatment of myocardial infarction. Examples include angiogenesis gene therapy (82), proangiogenic stem cell therapy (83), angiogenic ncRNA therapy (84), and exosome- and exosome-derived proangiogenic ncRNA therapy (85-88). For example, a study by Ma et al. showed that exosomes derived from murine BMSCs can deliver MIR132, induce tube formation in HUVECs in vitro, and promote angiogenesis in the ischemic area of the infarcted heart in mice (89).

Exosomal lncRNAs derived from stem cells can promote endothelial cell proliferation and angiogenesis after myocardial infarction. For example, Q. Wu et al. found that hypoxia-induced cardiovascular progenitor cells derived from human pluripotent stem cell (hCVPC)-EVs could improve heart function in mice with myocardial infarction, and immunohistochemistry studies showed that CD31 and $\alpha$-SMA expression increased. Similarly, in vitro experiments showed that hCVPC-EVs can reduce the damage induced by oxygen glucose deprivation (OGD) to cardiomyocytes and promote endothelial cell migration and tube formation. RNA sequencing and RT-qPCR analyses showed that the expression of MALAT1 in hypoxic hCVPC-EVs was significantly increased, and knocking down the expression of MALAT1 inhibited angiogenesis. Database and dual luciferase gene reporter assays suggest that MALAT1 targets MIR497 (44). After culturing atorvastatin-treated (ATV)-MSC-exos with HUVECs in vitro, Huang et al. showed that HUVECs treated with ATV-MSC-exos had enhanced tube-forming abilities. When cocultured with H9C2 cardiomyocytes stimulated with hypoxia and serum deprivation (H/SD), HUVEC-exos increased the survival rates of cardiomyocytes under H/SD conditions and inhibited myocardial fibrosis. In addition, rats with myocardial infarction were injected with ATV-MSC-exos, resulting in improved heart function, inhibited cardiomyocyte apoptosis, reduced inflammatory cell infiltration, and increased blood vessel density. The sequencing of ATV-MSC-exos demonstrated that the lncRNA H19 was highly expressed, and the protective effect of ATV-MSC-exos on cardiomyocytes and the promotion of endothelial cell angiogenesis were eliminated after $H 19$ knockout. Furthermore, MIR-675-3p, MIR-675-5p, VEGF, and ICAM1 were positively expressed with $H 19$. These results suggest that atorvastatin increases the secretion of $\mathrm{H} 19$ exosomes by rat mesenchymal cells, activates VEGF and ICAM1 by regulating the expression of $M I R-675-3 p$ and $M I R-675-5 p$, and promotes angiogenesis in the endothelium after myocardial infarction (59).

Studies have shown that $\mathrm{HBO}$ can promote the expression of angiogenic exosomal lncRNAs and mediate angiogenesis after myocardial infarction. For example, Shyu et al. found that HBOinduced cardiomyocyte exosomes increased the expression of MALAT1, decreased the expression of MIR92A, increased the expression of KLF2 and CD31, reduced the area of myocardial infarction and inhibited heart remodeling. A luciferase activity assay verified that the target gene of MIR92A was KLF2. In an in vitro rat cardiomyocyte hypoxia model, an $\mathrm{HBO}$ intervention promoted the expression of exosomal MALAT1 more than hypoxia stimulation. HBO induces the enrichment of exo-MALAT1 derived from cardiomyocytes, regulates the expression of CD31 and KLF2 through the MIR92A/KLF2 axis, and promotes cardiac angiogenesis after myocardial infarction (45). The metastasis of angiogenic lncRNAs in exosomes is of great significance for ischemic diseases. There have been many reports in the field of cancer. We look forward to more studies concerning angiogenesis in exosomal lncRNAs. It is believed that exosomal lncRNAs could be a new target for the treatment of ischemic cardiomyopathy.

\section{CARDIAC AGING}

Cellular aging is an irreversible factor in the occurrence and development of CVD. Aging leads to excessive oxidative stress, chronic low-grade inflammation, telomere shortening, autophagy, and mitochondrial dysfunction (90-92). Aging also mediates communication between cardiomyocytes and endothelial cells, fibroblasts, and immune cells (93) and promotes vascular wall endothelial damage, atherosclerosis, myocardial fibrosis, coronary heart disease, and heart failure (94, 95). Currently, the strategies applied to delay cardiac aging include repairing mitochondrial dysfunction (96), targeting cardiac stem cell senescence and senescence-associated secretory phenotype changes (97), inducing autophagy (90), and hydrogen sulfidemediated regulation of senescence signals (98). It has been reported that extracellular vesicles mediate cell senescence (99). Recently, Lei et al. found that the transfer of proliferating cell nuclear antigen from MSC-EVs derived from neonatal umbilical cords could reverse aging in adult BMSCs. In mice, UCMSCEVs could delay the aging phenotype in aging mice and reduce degeneration in bones and kidneys (100).

Recent studies have shown that stem cell exosomal lncRNAs can combat myocardial aging. For example, Zhu et al. injected hUCMSC-exo-MALAT1 into the tail veins of aging mice and found that the UCMSC-exos reversed the adverse effects of aging on cardiac function, increased the mRNA expression of the cardiac antiaging marker TERT, reduced the mRNA expression of the cardiac aging marker $P 21$ and the inflammatory factor TNF- $\alpha$, and reduced the protein expression of p-p65 in cardiac tissue, but these antiaging effects were reversed by siMALAT1. Culturing UCMSC-exo-MALAT1 with senescent cardiomyocytes reduced the activity of $N F-\kappa B$ and the expression levels of p-p65 and $T N F-\alpha$, but silencing MALAT1 blocked these effects. These studies suggest that UCMSC-exos can fight cardiac aging through the MALAT1/NF- $\kappa B / T N F-\alpha$ axis (46). Similarly, Xia showed that adipose MSC (ADMSC)-derived exosomes were enriched with MALAT1 under hypoxic conditions. Hypoxic MSC-exos were cultured with cardiomyocytes and inhibited doxorubicin (Dox)-induced cardiomyocyte senescence. In addition, hypoxic MSCs-exos can improve myocardial cell metabolism disorders. 
Database and luciferase reporter assays show that hypoxiainduced ADMSCs-exo-MALAT1 can improve mitochondrial dysfunction in cardiomyocytes after Dox treatment by targeting MIR-92a-3p/ATG4A and combating cardiac aging (47). In addition, Zhuang et al. found that the lncRNA NEAT1 was significantly enriched in exosomes derived from BMSCs treated with MIF. MSCs ${ }^{\text {MIF }}$-exos can resist Dox-induced myocardial aging in mice and improve heart function. In cell experiments, MSCs $^{\text {MIF }}$-exos could also resist Dox-induced senescence in cardiomyocytes by reducing the number of cells in the G0/G1 phase, reducing the expression of the senescence genes P27 and $P 16$, reducing the percentage of SA- $\beta$-gal-positive cells, and increasing the telomere length and activity. Database and luciferase reporter assays show that $\mathrm{MSCs}^{\mathrm{MIF}}$-exos regulate the MIR-221-3p/SIRT2 axis by transporting NEAT1 to protect against Dox-induced cardiomyocyte senescence (52). We agree that stem cells are regarded as a breakthrough point against cell senescence, and the results of exosomal lncRNAs derived from stem cells against cardiomyocyte senescence are encouraging. Furthermore, exosomal lncRNAs provide a new perspective for the treatment of cell senescence and cell metabolic damage.

\section{OTHER CARDIOVASCULAR DISEASES}

Wang et al. found exosomal lncRNA crosstalk in chronic kidney disease (CKD) and CVD. The authors showed an increased expression of the lncRNA ZFAS1 in the hearts of CKD mice, transfected ZFAS1 into human cardiomyocytes (HCMs) and collected exosomes, and found that ZFAS1 was significantly enriched in HCM-derived exosomes. Injection of HCM-exos into mice significantly reduced their heart function and induced myocardial fibrosis. Similarly, HCM-exos activated the $\mathrm{WNT} / \beta$-catenin signaling pathway in mouse cardiomyocytes after intervention in vitro. Silencing ZFAS1 can inhibit fibrosis in human CFs (HCFs). Biological database and luciferase assays show that ZFAS1 is transferred to HCFs through exosomes secreted by HCMs and induces myocardial fibrosis through the $M I R-4711-5 p / \mathrm{WNT} / \beta$ signaling pathway (60). The communication of exosomal lncRNAs between CKD and CVD provides a reference for the study of the relationship between other systemic diseases and CVD.

Luo et al. found 105 significantly upregulated lncRNAs, 126 significantly downregulated lncRNAs, 77 significantly upregulated mRNAs, and 102 significantly downregulated mRNAs in plasma exosomes from five patients with rheumatic mitral stenosis. GO and KEGG analyses of the differentially expressed lncRNA-related genes showed that these cells are involved in magnesium homeostasis, ERBB signaling, RAS signaling, and inflammation. The KEGG pathway analysis of the upregulated mRNAs showed that they are associated with pathways associated with hypertrophic cardiomyopathy and dilated cardiomyopathy. In addition, the analysis of differentially expressed lncRNA subgroups indicated that five pairs of lncRNAs and their accessory-related genes were coexpressed simultaneously; the four downregulated pairs were RP13820C6.2/EP400, RP11-339B21.15/CERCAM, G004800/ZBTB7B,
XLOC_010028/PDE3A, and the one downregulated pair was XLOC_004201/STOX2. This finding highlights the potential diagnostic and therapeutic role of exosomal lncRNAs in rheumatic heart disease (65).

\section{OUTLOOK AND SUMMARY}

Current research concerning exosomes and exosomal lncRNAs is still in its infancy. First, the current methods used for the separation and purification of exosomes are different, and the production capacity is low, which is far from the standard of clinical application. It is of practical significance to upgrade the technology used for the separation and purification of exosomes. For example, Chen et al. optimized the yield and purity of exosomes by using an ultrafast separation system (EXODUS), which was innovated by ultrafiltration (101). We suggest that the separation and purification of exosomes should achieve a high a standard as possible.

Second, the organ targeting of exosomes needs to be examined. Local injection into a target organ may have improved therapeutic effects. However, intravenous injection results in unsatisfactory retention rates of exosomes in the target organ. We agree that the targeting of organs can be increased by surface modification of engineered exosomes, but the low immunogenicity and non-tumorigenicity of exosomes should be retained (102). In addition, heterogeneity is a difficult problem in the study of exosomes (103). The biological and functional mechanisms of serum exosomal lncRNAs from different CVD patients and exosomal lncRNAs secreted by different cells are different. Therefore, we suggest that the separation and purification of exosomes should be carried out under uniform and appropriate standards to the greatest extent possible. Therefore, we should search for traces of exosomes and exosomal lncRNAs in serum or tissues from more CVD patients and perform functional analyses. Furthermore, the exploration of the interaction between cells from different sources and target cells of exosomal lncRNAs should be more groundbreaking. Finally, deciphering the specific molecular mechanism of lncRNA selection and modification in exosomes may introduce new perspectives.

In addition, the relationship between lncRNAs and exosomes is intriguing. In general, exosomes act as carriers of lncRNAs to achieve cell-to-cell transfer and regulation. However, Yang et al. found that increasing the level of the lncRNA HOTAIR can promote the secretion of hepatocellular carcinoma exosomes (104). Similarly, Xing et al. found that the lncRNA HAND2$A S 1$ can inhibit the level of exo-MIR-106a-5p secreted by MSCs (105). In the field of CVD, research by $\mathrm{Li}$ showed that the overexpression of the IncRNA NRON can significantly inhibit the expression of exo-MIR23A secreted by atrial myocytes, promote the polarization of M2 macrophages, and reduce atrial fibrosis (106). The above studies show that lncRNAs, exosomes and lncRNAs and miRNAs in exosomes are closely related and complex. Therefore, we believe that in the CVD field, more attention and action are still needed in the study of the 
interaction mechanism among exosomes and exosomal lncRNAs and miRNAs.

The application and innovation of engineered exosomes may compensate for the disadvantage of exosomes produced by natural cells. Gene-enriched exosomes can be obtained through physical and chemical approaches, such as electroporation, ultrasound, and liposome-mediated membrane fusion (107). The surface modification of engineered exosomes allows exosomes to target tissues or organs and can be used to monitor the pharmacokinetics of exosomes in the body in real time to produce ideal biological effects (102). Therefore, using the advantages of engineered exosomes can provide reveries for the therapeutic effects of exosomal lncRNAs in the clinical application of real CVD patients. Of course, these applications can only be applied after solving the heterogeneity of exosomal lncRNAs and satisfying their basic conditions as drug carriers. In addition, proteomic and lipidomic studies of exosomes are potential hotspots in the study of CVD. For example, Takov et al. conducted a proteomic analysis of EVs derived from MSCs and found that migration-promoting mediators, such as

\section{REFERENCES}

1. Roth GA, Mensah GA, Johnson CO, Addolorato G, Ammirati E, Baddour LM, et al. Global burden of cardiovascular diseases and risk factors, 19902019: update from the GBD 2019 study. J Am Coll Cardiol. (2020) 76:29823021. doi: 10.1016/j.jacc.2020.11.010

2. Wu Q, Bai B, Tian C, Li D, Yu H, Song B, et al. The molecular mechanisms of cardiotoxicity induced by HER2, VEGF, and tyrosine kinase inhibitors: an updated review. Cardiovasc Drugs Therapy. (2021). doi: 10.1007/s10557-021-07181-3. [Epub ahead of print].

3. Lee H, Zhang Z, Krause HM. Long noncoding RNAs and repetitive elements: junk or intimate evolutionary partners? Trends Genet. (2019) 35:892902. doi: 10.1016/j.tig.2019.09.006

4. Palazzo AF, Koonin EV. Functional long non-coding RNAs evolve from junk transcripts. Cell. (2020) 183:1151-61. doi: 10.1016/j.cell.2020.09.047

5. Zhang $\mathrm{C}$, Han $\mathrm{B}, \mathrm{Xu} \mathrm{T}, \mathrm{Li} \mathrm{D}$. The biological function and potential mechanism of long non-coding RNAs in cardiovascular disease. J Cell $\mathrm{Mol}$ Med. (2020) 24:12900-9. doi: 10.1111/jcmm.15968

6. Lou W, Ding B, Fu P. Pseudogene-derived lncRNAs and their miRNA sponging mechanism in human cancer. Front Cell Dev Biol. (2020) 8:85. doi: $10.3389 /$ fcell.2020.00085

7. Raposo G, Stoorvogel W. Extracellular vesicles: exosomes, microvesicles, and friends. J Cell Biol. (2013) 200:373-83. doi: 10.1083/jcb.201211138

8. Zhou R, Chen KK, Zhang J, Xiao B, Huang Z, Ju C, et al. The decade of exosomal long RNA species: an emerging cancer antagonist. Mol Cancer. (2018) 17:75. doi: 10.1186/s12943-018-0823-Z

9. Zhang WL, Liu Y, Jiang J, Tang YJ, Tang YL, Liang XH. Extracellular vesicle long non-coding RNA-mediated crosstalk in the tumor microenvironment: tiny molecules, huge roles. Cancer Sci. (2020) 111:2726-35. doi: 10.1111/cas.14494

10. Statello L, Guo CJ, Chen LL, Huarte M. Gene regulation by long noncoding RNAs and its biological functions. Nat Rev Mol Cell Biol. (2021) 22:96-118. doi: 10.1038/s41580-020-00315-9

11. Chen LL. Linking long noncoding RNA localization and function. Trends Biochem Sci. (2016) 41:761-72. doi: 10.1016/j.tibs.2016.07.003

12. Xu ZM, Huang F, Huang WQ. Angiogenic lncRNAs: a potential therapeutic target for ischaemic heart disease. Life Sci. (2018) 211:15771. doi: 10.1016/j.lfs.2018.09.022

13. Quinn JJ, Chang HY. Unique features of long non-coding RNA biogenesis and function. Nat Rev Genet. (2016) 17:47-62. doi: 10.1038/nrg.2015.10
PTX3, BGN, and RTN4, mediate angiogenesis after myocardial infarction (108). In summary, we believe that exosomes and exosomal lncRNAs may play an important role in the diagnosis and treatment of CVD in the future.

\section{AUTHOR CONTRIBUTIONS}

ZY wrote the manuscript, figure legends, and created the figures and tables. WH revised the manuscript. All authors contributed to the article and approved the submitted version.

\section{FUNDING}

The present study was funded by the National Natural Science Foundation of China (Grant No. 81960056).

\section{ACKNOWLEDGMENTS}

The authors thank all of the individuals who participated in the investigations.
14. Kopp F, Mendell JT. Functional classification and experimental dissection of long noncoding RNAs. Cell. (2018) 172:393407. doi: 10.1016/j.cell.2018.01.011

15. Yao RW, Wang Y, Chen LL. Cellular functions of long noncoding RNAs. Nat Cell Biol. (2019) 21:542-51. doi: 10.1038/s41556-019-0311-8

16. Jain AK, Xi Y, McCarthy R, Allton K, Akdemir KC, Patel LR, et al. LncPRESS1 Is a p53-regulated LncRNA that safeguards pluripotency by disrupting SIRT6-mediated de-acetylation of histone H3K56. Mol Cell. (2016) 64:96781. doi: 10.1016/j.molcel.2016.10.039

17. Latos PA, Pauler FM, Koerner MV, Senergin HB, Hudson QJ, Stocsits RR, et al. Airn transcriptional overlap, but not its lncRNA products, induces imprinted Igf2r silencing. Science. (2012) 338:1469-72. doi: 10.1126/science.1228110

18. Abels ER, Breakefield XO. Introduction to extracellular vesicles: biogenesis, RNA Cargo selection, content, release, and uptake. Cell Mol Neurobiol. (2016) 36:301-12. doi: 10.1007/s10571-016-0366-z

19. Teng F, Fussenegger M. Shedding light on extracellular vesicle biogenesis and bioengineering. Adv Sci. (2020) 8:2003505. doi: 10.1002/advs.202003505

20. D'Souza-Schorey C, Schorey JS. Regulation and mechanisms of extracellular vesicle biogenesis and secretion. Essays Biochem. (2018) 62:125-33. doi: 10.1042/EBC20170078

21. Stuffers S, Sem Wegner C, Stenmark H, Brech A. Multivesicular endosome biogenesis in the absence of ESCRTs. Traffic. (2009) 10:92537. doi: 10.1111/j.1600-0854.2009.00920.x

22. Xu R, Rai A, Chen M, Suwakulsiri W, Greening DW, Simpson RJ. Extracellular vesicles in cancer - implications for future improvements in cancer care. Nat Rev Clin Oncol. (2018) 15:617-38. doi: 10.1038/s41571-018-0036-9

23. Tian C, Yang Y, Bai B, Wang S, Liu M, Sun RC, et al. Potential of exosomes as diagnostic biomarkers and therapeutic carriers for doxorubicin-induced cardiotoxicity. Int J Biol Sci. (2021) 17:1328-38. doi: 10.7150/ijbs.58786

24. Ghafarian F, Pashirzad M, Khazaei M, Rezayi M, Hassanian SM, Ferns GA, et al. The clinical impact of exosomes in cardiovascular disorders: from basic science to clinical application. J Cell Physiol. (2019) 234:1222636. doi: $10.1002 /$ jcp. 27964

25. Lei Y, Guo W, Chen B, Chen L, Gong J, Li W. Tumor-released lncRNA H19 promotes gefitinib resistance via packaging into exosomes in non-small cell lung cancer. Oncol Rep. (2018) 40:3438-46. doi: 10.3892/or.2018.6762

26. Han M, Gu Y, Lu P, Li J, Cao H, Li X, et al. Exosome-mediated lncRNA AFAP1-AS1 promotes trastuzumab resistance through binding 
with AUF1 and activating ERBB2 translation. Mol Cancer. (2020) 19:26. doi: 10.1186/s12943-020-1145-5

27. Ahadi A, Brennan S, Kennedy PJ, Hutvagner G, Tran N. Long non-coding RNAs harboring miRNA seed regions are enriched in prostate cancer exosomes. Sci Rep. (2016) 6:24922. doi: 10.1038/srep24922

28. Gao T, Liu X, He B, Nie Z, Zhu C, Zhang P, et al. Exosomal lncRNA $91 \mathrm{H}$ is associated with poor development in colorectal cancer by modifying HNRNPK expression. Cancer Cell Int. (2018) 18:11. doi: 10.1186/s12935-018-0506-2

29. Deng X, Ruan H, Zhang X, Xu X, Zhu Y, Peng H, et al. Long noncoding RNA CCAL transferred from fibroblasts by exosomes promotes chemoresistance of colorectal cancer cells. Int J Cancer. (2020) 146:170016. doi: 10.1002/ijc. 32608

30. Fabbiano F, Corsi J, Gurrieri E, Trevisan C, Notarangelo M, D’Agostino VG. RNA packaging into extracellular vesicles: an orchestra of RNA-binding proteins? J Extracell Vesicles. (2020) 10:e12043. doi: 10.1002/jev2.12043

31. Qu L, Ding J, Chen C, Wu ZJ, Liu B, Gao Y, et al. Exosometransmitted lncARSR promotes sunitinib resistance in renal cancer by acting as a competing endogenous RNA. Cancer Cell. (2016) 29:65368. doi: 10.1016/j.ccell.2016.03.004

32. Zheng D, Huo M, Li B, Wang W, Piao H, Wang Y, et al. The role of exosomes and exosomal microRNA in cardiovascular disease. Front Cell Dev Biol. (2020) 8:616161. doi: 10.3389/fcell.2020.616161

33. Zang X, Gu J, Zhang J, Shi H, Hou S, Xu X, et al. Exosome-transmitted IncRNA UFC1 promotes non-small-cell lung cancer progression by EZH2mediated epigenetic silencing of PTEN expression. Cell Death Dis. (2020) 11:215. doi: 10.1038/s41419-020-2409-0

34. Zhuo C, Yi T, Pu J, Cen X, Zhou Y, Feng S, et al. Exosomal linc-FAM138B from cancer cells alleviates hepatocellular carcinoma progression via regulating miR-765. Aging. (2020) 12:26236-47. doi: 10.18632/aging.202430

35. Xie Y, Dang W, Zhang S, Yue W, Yang L, Zhai X, et al. The role of exosomal noncoding RNAs in cancer. Mol Cancer. (2019) 18:37. doi: 10.1186/s12943-019-0984-4

36. Li Y, Yin Z, Fan J, Zhang S, Yang W. The roles of exosomal miRNAs and lncRNAs in lung diseases. Signal Transduct Target Therapy. (2019) 4:47. doi: 10.1038/s41392-019-0080-7

37. Wang Y, Zhao R, Shen C, Liu W, Yuan J, Li C, et al. Exosomal CircHIPK3 released from hypoxia-induced cardiomyocytes regulates cardiac angiogenesis after myocardial infarction. Oxidat Med Cell Longevity. (2020) 2020:8418407. doi: 10.1155/2020/8418407

38. Wang Y, Zhao R, Liu W, Wang Z, Rong J, Long X, et al. Exosomal circHIPK3 released from hypoxia-pretreated cardiomyocytes regulates oxidative damage in cardiac microvascular endothelial cells via the miR-29a/IGF-1 pathway. Oxid Med Cell Longev. (2019) 2019:7954657. doi: 10.1155/2019/7954657

39. Ni YQ, Lin X, Zhan JK, Liu YS. Roles and functions of exosomal non-coding RNAs in vascular aging. Aging Dis. (2020) 11:164-78. doi: 10.14336/AD.2019.0402

40. Chen D, Wang K, Zheng Y, Wang G, Jiang M. Exosomes-mediated LncRNA ZEB1-as1 facilitates cell injuries by mir-590-5p/ETS1 axis through the TGF- $\beta$ /smad pathway in oxidized low-density lipoprotein-induced human umbilical vein endothelial cells. J Cardiovasc Pharmacol. (2021) 77:48090. doi: 10.1097/FJC.0000000000000974

41. Shan K, Jiang Q, Wang XQ, Wang YN, Yang H, Yao MD, et al. Role of long non-coding RNA-RNCR3 in atherosclerosis-related vascular dysfunction. Cell Death Dis. (2016) 7:e2248. doi: 10.1038/cddis.2016.145

42. Li H, Zhu X, Hu L, Li Q, Ma J, Yan J. Loss of exosomal MALAT1 from ox-LDL-treated vascular endothelial cells induces maturation of dendritic cells in atherosclerosis development. Cell Cycle (Georgetown, Tex). (2019) 18:2255-67. doi: 10.1080/15384101.2019.1642068

43. Huang C, Han J, Wu Y, Li S, Wang Q, Lin W, et al. Exosomal MALAT1 derived from oxidized low-density lipoprotein-treated endothelial cells promotes M2 macrophage polarization. Mol Med Rep. (2018) 18:50915. doi: $10.3892 / \mathrm{mmr} .2018 .8982$

44. Wu Q, Wang J, Tan WLW, Jiang Y, Wang S, Li Q, et al. Extracellular vesicles from human embryonic stem cell-derived cardiovascular progenitor cells promote cardiac infarct healing through reducing cardiomyocyte death and promoting angiogenesis. Cell Death Dis. (2020) 11:354. doi: 10.1038/s41419-020-2508-y

45. Shyu KG, Wang BW, Fang WJ, Pan CM, Lin CM. Hyperbaric oxygeninduced long non-coding RNA MALAT1 exosomes suppress MicroRNA-92a expression in a rat model of acute myocardial infarction. J Cell Mol Med. (2020) 24:12945-54. doi: 10.1111/jcmm.15889

46. Zhu B, Zhang L, Liang C, Liu B, Pan X, Wang Y, et al. Stem cellderived exosomes prevent aging-induced cardiac dysfunction through a novel exosome/lncRNA MALAT1/NF- $\mathrm{B} / \mathrm{TNF}-\alpha$ signaling pathway. Oxid Med Cell Longev. (2019) 2019:9739258. doi: 10.1155/2019/9739258

47. Xia W, Chen H, Xie C, Hou M. Long-noncoding RNA MALAT1 sponges microRNA-92a-3p to inhibit doxorubicin-induced cardiac senescence by targeting ATG4a. Aging. (2020) 12:8241-60. doi: 10.18632/aging.103136

48. Chen L, Yang W, Guo Y, Chen W, Zheng P, Zeng J, et al. Exosomal lncRNA GAS5 regulates the apoptosis of macrophages and vascular endothelial cells in atherosclerosis. PLoS ONE. (2017) 12:e0185406. doi: 10.1371/journal.pone.0185406

49. Song Y, Li H, Ren X, Li H, Feng C. SNHG9, delivered by adipocytederived exosomes, alleviates inflammation and apoptosis of endothelial cells through suppressing TRADD expression. Eur J Pharmacol. (2020) 872:172977. doi: 10.1016/j.ejphar.2020.172977

50. Zhang Z, Yi D, Zhou J, Zheng Y, Gao Z, Hu X, et al. Exosomal LINC01005 derived from oxidized low-density lipoprotein-treated endothelial cells regulates vascular smooth muscle cell phenotypic switch. BioFactors. (2020) 46:743-53. doi: 10.1002/biof. 1665

51. Chen H, Xia W, Hou M. LncRNA-NEAT1 from the competing endogenous RNA network promotes cardioprotective efficacy of mesenchymal stem cell-derived exosomes induced by macrophage migration inhibitory factor via the miR-142-3p/FOXO1 signaling pathway. Stem Cell Res Ther. (2020) 11:31. doi: 10.1186/s13287-020-1556-7

52. Zhuang L, Xia W, Chen D, Ye Y, Hu T, Li S, et al. Exosomal LncRNANEAT1 derived from MIF-treated mesenchymal stem cells protected against doxorubicin-induced cardiac senescence through sponging miR-221-3p. $J$ Nanobiotechnol. (2020) 18:157. doi: 10.1186/s12951-020-00716-0

53. Wang L, Zhang J. Exosomal lncRNA AK139128 derived from hypoxic cardiomyocytes promotes apoptosis and inhibits cell proliferation in cardiac fibroblasts. Int J Nanomedicine. (2020) 15:3363-76. doi: 10.2147/IJN.S240660

54. Mao Q, Liang XL, Zhang CL, Pang YH, Lu YX. LncRNA KLF3-AS1 in human mesenchymal stem cell-derived exosomes ameliorates pyroptosis of cardiomyocytes and myocardial infarction through miR-138-5p/Sirt1 axis. Stem Cell Res Ther. (2019) 10:393. doi: 10.1186/s13287-019-1522-4

55. Zhai TY, Cui BH, Zhou Y, Xu XY, Zou L, Lin X, et al. Exosomes released from CaSR-stimulated PMNs reduce ischaemia/reperfusion injury. Oxid Med Cell Longev. (2021) 2021:3010548. doi: 10.1155/2021/3010548

56. Diao L, Zhang Q. Transfer of IncRNA UCAl by hUCMSCs-derived exosomes protects against hypoxia/reoxygenation injury through impairing miR-143-targeted degradation of Bcl-2. Aging. (2021) 13:202520. doi: 10.18632/aging.202520

57. Sun L, Zhu W, Zhao P, Wang Q, Fan B, Zhu Y, et al. Long noncoding RNA UCA1 from hypoxia-conditioned hMSC-derived exosomes: a novel molecular target for cardioprotection through miR-873-5p/XIAP axis. Cell Death Dis. (2020) 11:696. doi: 10.1038/s41419-020-02783-5

58. Su Q, Lv XW, Xu YL, Cai RP, Dai RX, Yang XH, et al. Exosomal LINC00174 derived from vascular endothelial cells attenuates myocardial I/R injury via p53-mediated autophagy and apoptosis. Mol Ther Nucl Acids. (2021) 23:1304-22. doi: 10.1016/j.omtn.2021.02.005

59. Huang $\mathrm{P}$, Wang L, Li Q, Tian X, Xu J, Xu J, et al. Atorvastatin enhances the therapeutic efficacy of mesenchymal stem cells-derived exosomes in acute myocardial infarction via up-regulating long noncoding RNA H19. Cardiovasc Res. (2020) 116:353-67. doi: 10.1093/cvr/ cvz139

60. Wang Y, Cao X, Yan L, Zheng Y, Yu J, Sun F, et al. Exosome-derived long non-coding RNA ZFAS1 controls cardiac fibrosis in chronic kidney disease. Aging. (2021) 13:202599. doi: 10.18632/aging.202599

61. Wang Y, Liang J, Xu J, Wang X, Zhang X, Wang W, et al. Circulating exosomes and exosomal IncRNA HIF1A-AS1 in atherosclerosis. Int J Clin Exp Pathol. (2017) 10:8383-8. 
62. Chen Z, Yan Y, Wu J, Qi C, Liu J, Wang J. Expression level and diagnostic value of exosomal NEAT1/miR-204/MMP-9 in acute STsegment elevation myocardial infarction. IUBMB Life. (2020) 72:2499507. doi: 10.1002/iub.2376

63. Zheng ML, Liu XY, Han RJ, Yuan W, Sun K, Zhong JC, et al. Circulating exosomal long non-coding RNAs in patients with acute myocardial infarction. J Cell Mol Med. (2020) 24:9388-96. doi: 10.1111/jcmm.15589

64. Liang C, Zhang L, Lian X, Zhu T, Zhang Y, Gu N. Circulating exosomal SOCS2-AS1 acts as a novel biomarker in predicting the diagnosis of coronary artery disease. Biomed Res Int. (2020) 2020:9182091. doi: 10.1155/2020/9182091

65. Luo Y, Huang L, Luo W, Ye S, Hu Q. Genomic analysis of lncRNA and mRNA profiles in circulating exosomes of patients with rheumatic heart disease. Biol Open. (2019) 8:bio045633. doi: 10.1242/bio.045633

66. Hergenreider E, Heydt S, Tréguer K, Boettger T, Horrevoets AJ, Zeiher $\mathrm{AM}$, et al. Atheroprotective communication between endothelial cells and smooth muscle cells through miRNAs. Nat Cell Biol. (2012) 14:24956. doi: $10.1038 /$ ncb 2441

67. Bouchareychas L, Duong P, Covarrubias S, Alsop E, Phu TA, Chung A, et al. Macrophage exosomes resolve atherosclerosis by regulating hematopoiesis and inflammation via MicroRNA Cargo. Cell Rep. (2020) 32:107881. doi: 10.1016/j.celrep.2020.107881

68. Neeland IJ, Ross R, Després JP, Matsuzawa Y, Yamashita S, Shai I, et al. Visceral and ectopic fat, atherosclerosis, and cardiometabolic disease: a position statement. Lancet Diabet Endocrinol. (2019) 7:71525. doi: 10.1016/S2213-8587(19)30084-1

69. Rocha VZ, Libby P. Obesity, inflammation, and atherosclerosis. Nat Rev Cardiol. (2009) 6:399-409. doi: 10.1038/nrcardio.2009.55

70. Huang $\mathrm{K}, \mathrm{Hu} \mathrm{S}$, Cheng $\mathrm{K}$. A new era of cardiac cell therapy: opportunities and challenges. Adv Healthc Mater. (2019) 8:e1801011. doi: 10.1002/adhm.201801011

71. Li Z, Hu S, Cheng K. Chemical engineering of cell therapy for heart diseases. Acc Chem Res. (2019) 52:1687-96. doi: 10.1021/acs.accounts.9b00137

72. Spannbauer A, Mester-Tonczar J, Traxler D, Kastner N, Zlabinger K, Hašimbegović E, et al. Large animal models of cell-free cardiac regeneration. Biomolecules. (2020) 10:1392. doi: 10.3390/biom10101392

73. Tan SJO, Floriano JF, Nicastro L, Emanueli C, Catapano F. Novel applications of mesenchymal stem cell-derived exosomes for myocardial infarction therapeutics. Biomolecules. (2020) 10:707. doi: 10.3390/biom10050707

74. Saludas L, Oliveira CC, Roncal C, Ruiz-Villalba A, Prósper F, Garbayo E, et al. Extracellular vesicle-based therapeutics for heart repair. Nanomaterials (Basel, Switzerland). (2021) 11:570. doi: 10.3390/nano11030570

75. Khan M, Nickoloff E, Abramova T, Johnson J, Verma SK, Krishnamurthy P, et al. Embryonic stem cell-derived exosomes promote endogenous repair mechanisms and enhance cardiac function following myocardial infarction. Circ Res. (2015) 117:52-64. doi: 10.1161/CIRCRESAHA.117.305990

76. Gallet R, Dawkins J, Valle J, Simsolo E, de Couto G, Middleton R, et al. Exosomes secreted by cardiosphere-derived cells reduce scarring, attenuate adverse remodelling, and improve function in acute and chronic porcine myocardial infarction. Eur Heart J. (2017) 38:20111. doi: 10.1093/eurheartj/ehw240

77. Fröhlich GM, Meier P, White SK, Yellon DM, Hausenloy DJ. Myocardial reperfusion injury: looking beyond primary PCI. Eur Heart J. (2013) 34:1714-22. doi: 10.1093/eurheartj/eht090

78. Hausenloy DJ, Yellon DM. Ischaemic conditioning and reperfusion injury. Nat Rev Cardiol. (2016) 13:193-209. doi: 10.1038/nrcardio.2016.5

79. Hausenloy DJ, Yellon DM. Myocardial ischemia-reperfusion injury: a neglected therapeutic target. J Clin Investigat. (2013) 123:92-100. doi: 10.1172/JCI62874

80. Røsand $\varnothing$, Høydal MA. Cardiac exosomes in ischemic heart disease- a narrative review. Diagnostics (Basel, Switzerland). (2021) 11:269. doi: 10.3390/diagnostics11020269

81. Luo $\mathrm{H}, \mathrm{Li} \mathrm{X}, \mathrm{Li} \mathrm{T}$, Zhao L, He J, Zha L, et al. microRNA-423-3p exosomes derived from cardiac fibroblasts mediates the cardioprotective effects of ischaemic post-conditioning. Cardiovasc Res. (2019) 115:1189204. doi: $10.1093 / \mathrm{cvr} / \mathrm{cvy} 231$
82. Ylä-Herttuala S, Bridges C, Katz MG, Korpisalo P. Angiogenic gene therapy in cardiovascular diseases: dream or vision? Eur Heart J. (2017) 38:136571. doi: 10.1093/eurheartj/ehw547

83. Wang Z, Su X, Ashraf M, Kim IM, Weintraub NL, Jiang M, et al. Regenerative therapy for cardiomyopathies. J Cardiovasc Transl Res. (2018) 11:35765. doi: 10.1007/s12265-018-9807-z

84. Yu B, Wang S. Angio-LncRs: LncRNAs that regulate angiogenesis and vascular disease. Theranostics. (2018) 8:3654-75. doi: 10.7150/thno.26024

85. Johnson T, Zhao L, Manuel G, Taylor H, Liu D. Approaches to therapeutic angiogenesis for ischemic heart disease. J Mol Med. (2019) 97:14151. doi: 10.1007/s00109-018-1729-3

86. Kesidou D, da Costa Martins PA, de Windt LJ, Brittan M, Beqqali A, Baker AH. Extracellular vesicle miRNAs in the promotion of cardiac neovascularisation. Front Physiol. (2020) 11:579892. doi: 10.3389/fphys.2020.579892

87. Witman N, Zhou C, Grote Beverborg N, Sahara M, Chien KR. Cardiac progenitors and paracrine mediators in cardiogenesis and heart regeneration. Semin Cell Dev Biol. (2020) 100:2951. doi: 10.1016/j.semcdb.2019.10.011

88. Moghiman T, Barghchi B, Esmaeili SA, Shabestari MM, Tabaee SS, MomtaziBorojeni AA. Therapeutic angiogenesis with exosomal microRNAs: an effectual approach for the treatment of myocardial ischemia. Heart Failure Rev. (2020) 26:205-13. doi: 10.1007/s10741-020-10001-9

89. Ma T, Chen Y, Chen Y, Meng Q, Sun J, Shao L, et al. MicroRNA132, delivered by mesenchymal stem cell-derived exosomes, promote angiogenesis in myocardial infarction. Stem Cells Int. (2018) 2018:3290372. doi: 10.1155/2018/3290372

90. Miyamoto S. Autophagy and cardiac aging. Cell Death Differ. (2019) 26:65364. doi: 10.1038/s41418-019-0286-9

91. Liang WJ, Gustafsson $\AA$ B. The aging heart: mitophagy at the center of rejuvenation. Front Cardiovasc Med. (2020) 7:18. doi: $10.3389 /$ fcvm.2020.00018

92. Quan Y, Xin Y, Tian G, Zhou J, Liu X. Mitochondrial ROS-modulated mtDNA: a potential target for cardiac aging. Oxidat Med Cell Longev. (2020) 2020:9423593. doi: 10.1155/2020/9423593

93. Tang X, Li PH, Chen HZ. Cardiomyocyte senescence and cellular communications within myocardial microenvironments. Front Endocrinol. (2020) 11:280. doi: 10.3389/fendo.2020.00280

94. Triposkiadis F, Xanthopoulos A, Butler J. Cardiovascular aging and heart failure: JACC review topic of the week. J Am Coll Cardiol. (2019) 74:80413. doi: 10.1016/j.jacc.2019.06.053

95. Dong M, Yang Z, Fang H, Xiang J, Xu C, Zhou Y, et al. Aging attenuates cardiac contractility and affects therapeutic consequences for myocardial infarction. Aging Dis. (2020) 11:365-76. doi: 10.14336/AD.2019.0522

96. Yeh $\mathrm{CH}$, Chou YJ, Kao CH, Tsai TF. Mitochondria and calcium homeostasis: Cisd2 as a big player in cardiac ageing. Int J Mol Sci. (2020) 21:9238. doi: 10.3390/ijms21239238

97. Cianflone E, Torella M, Biamonte F, De Angelis A, Urbanek K, Costanzo FS, et al. Targeting cardiac stem cell senescence to treat cardiac aging and disease. Cells. (2020) 9:1558. doi: 10.3390/cells9061558

98. Testai L, Citi V, Martelli A, Brogi S, Calderone V. Role of hydrogen sulfide in cardiovascular ageing. Pharmacol Res. (2020) 160:105125. doi: 10.1016/j.phrs.2020.105125

99. Urbanelli L, Buratta S, Sagini K, Tancini B, Emiliani C. Extracellular vesicles as new players in cellular senescence. Int J Mol Sci. (2016) 17:1408. doi: 10.3390/ijms17091408

100. Lei Q, Gao F, Liu T, Ren W, Chen L, Cao Y, et al. Extracellular vesicles deposit PCNA to rejuvenate aged bone marrow-derived mesenchymal stem cells and slow age-related degeneration. Sci Transl Med. (2021) 13:aaz8697. doi: 10.1126/scitranslmed.aaz8697

101. Chen Y, Zhu Q, Cheng L, Wang Y, Li M, Yang Q, et al. Exosome detection via the ultrafast-isolation system: EXODUS. Nat Methods. (2021) 18:2128. doi: 10.1038/s41592-020-01034-x

102. Peng H, Ji W, Zhao R, Yang J, Lu Z, Li Y, et al. Exosome: a significant nano-scale drug delivery carrier. J Mater Chem B. (2020) 8:7591608. doi: 10.1039/D0TB01499K 
103. Zhang Y, Bi J, Huang J, Tang Y, Du S, Li P. Exosome: a review of its classification, isolation techniques, storage, diagnostic and targeted therapy applications. Int J Nanomed. (2020) 15:6917-34. doi: 10.2147/IJN.S264498

104. Yang L, Peng X, Li Y, Zhang X, Ma Y, Wu C, et al. Long noncoding RNA HOTAIR promotes exosome secretion by regulating RAB35 and SNAP23 in hepatocellular carcinoma. Mol Cancer. (2019) 18:78. doi: 10.1186/s12943-019-0990-6

105. Xing L, Tang X, Wu K, Huang X, Yi Y, Huan J. LncRNA HAND2AS1 suppressed the growth of triple negative breast cancer via reducing secretion of MSCs derived exosomal miR-106a-5p. Aging. (2020) 13:42436. doi: 10.18632 /aging. 202148

106. Li J, Zhang Q, Jiao H. LncRNA NRON promotes M2 macrophage polarization and alleviates atrial fibrosis through suppressing exosomal miR23a derived from atrial myocytes. J Formos Med Assoc. (2020) 120:1512-9. doi: 10.1016/j.jfma.2020.11.004

107. Wu P, Zhang B, Ocansey DKW, Xu W, Qian H. Extracellular vesicles: a bright star of nanomedicine.
Biomaterials. (2021) 269:120467. doi: 10.1016/j.biomaterials.2020. 120467

108. Takov K, He Z, Johnston HE, Timms JF, Guillot PV, Yellon DM, et al. Small extracellular vesicles secreted from human amniotic fluid mesenchymal stromal cells possess cardioprotective and promigratory potential. Basic Res Cardiol. (2020) 115:26. doi: 10.1007/s00395-020-0785-3

Conflict of Interest: The authors declare that the research was conducted in the absence of any commercial or financial relationships that could be construed as a potential conflict of interest.

Copyright $\odot 2021$ Yuan and Huang. This is an open-access article distributed under the terms of the Creative Commons Attribution License (CC BY). The use, distribution or reproduction in other forums is permitted, provided the original author(s) and the copyright owner(s) are credited and that the original publication in this journal is cited, in accordance with accepted academic practice. No use, distribution or reproduction is permitted which does not comply with these terms. 